\title{
Eisenhart-Duval lift for minisuperspace quantum cosmology
}

\author{
Nahomi Kan* \\ National Institute of Technology, Gifu College, Motosu-shi, Gifu 501-0495, Japan \\ Takuma Aoyama, ${ }^{\dagger}$ Taiga Hasegawa, ${ }^{\ddagger}$ and Kiyoshi Shiraishi ${ }^{\S}$ \\ Graduate School of Sciences and Technology for Innovation, \\ Yamaguchi University, Yamaguchi-shi, Yamaguchi 753-8512, Japan
}

(Dated: October 6, 2021)

\begin{abstract}
We study covariant equations in quantum cosmology of an extended minisuperspace obtained by the Eisenhart-Duval lift. We find that a Dirac-type equation is naturally introduced in the extended minisuperspace. Explicit forms of the fundamental solutions are yielded for specific models. The possible further development in this direction is also discussed.
\end{abstract}

PACS numbers: 04.60.-m, 11.30.-j, 45.20.Jj, 98.80.Qc, 98.80.Jk.

\footnotetext{
* kan@gifu-nct.ac.jp

$\dagger$ b014vbv@yamaguchi-u.ac.jp

†019vbu@yamaguchi-u.ac.jp

§shiraish@yamaguchi-u.ac.jp
} 


\section{INTRODUCTION}

Quantum cosmology [1-5] has some problems to be solved. One of them is the process of deriving the probability density from the wave function of the universe. Since the usual Wheeler-DeWitt (WDW) equation in minisuperspace is a hyperbolic partial differential equation, it is very difficult to define a positive-definite probability density. Note that a component of the Klein-Gordon type conserved current may vanish if the wave function is substantially expressed by a real function, which often occurs as a solution of the WDW equation. Several hopeful approaches have been proposed to replace the WDW equation with other equations. For example, many authors have already proposed ideas to attempt a square root à la Dirac of the quadratic WDW equation [6-11], using two component wave functions [12], and applications of supersymmetric quantum mechanics [13-17], ${ }^{1}$ and the introduction of new "time" coordinates [19-23].

Another problem with quantum cosmology is the problem of factor ordering ${ }^{2}$ which arises with substitutions of operators for momenta [25-27]. In some toy models, a wave function with little difference can be obtained regardless of the choice in factor ordering of momentum operators to some extent. However, the choice is advocated to be important in some cases when considering various boundary conditions [28]. In particular, applying the Dirac square-root method mentioned above, it is also a considerable problem that the choice of ordering involves additional indefiniteness. In any case, when we wish to discuss some hidden mathematical structures and symmetry of gravitational theory minutely, we should not neglect the problem of factor ordering altogether.

Recently, remarkable papers [29, 30] have appeared that apply a type of the EisenhartDuval lift $[31,32]$, one of the classical methods in Hamiltonian dynamical systems [33-35] ${ }^{3}$ to cosmologies. ${ }^{4}$ In this Eisenhart's method, adding a dynamical variable, it is possible to describe a system by geometric treatment in the space of dynamical variables; that is, in the extended minisuperspace even in the presence of the potential term. Thus, the Hamiltonian of the system can be represented by the single Laplacian on the extended minisuperspace when the momenta are replaced by operators. It should be noted that the covariance in the

${ }^{1}$ For a review of supersymmetric quantum mechanics, please see Ref.[18].

2 Although the general factor-ordering problem in quantum gravity is known to be more difficult than the arrangement of momentum operators (see, for example, Ref. [24]) our present analysis is limited to the factor ordering on momentum operators.

3 See also a recent paper, Ref. [36].

4 See also Ref. [37, 38]. 
minisuperspace is not the general covariance of spacetime. The original idea of Eisenhart's work is to interpret a generic (nongeometrical) equation of motion as a (geometrical) geodesic equation in a space with a lifted metric.

We come to an idea that, if covariance in the extended minisuperspace is required as a guiding principle, the problem of factor ordering in the WDW equation disappears and it becomes possible to proceed with covariance as a prescription for Dirac square root.

In the present paper we consider the construction of covariant equations using the Eisenhart-Duval lift in minisuperspace quantum cosmology. For the sake of simplicity, we will focus on the case of a homogeneous and isotropic Friedmann-Lemaitre-RobertsonWalker (FLRW) universe containing a single spatially constant scalar field (that is, the case of two dynamical variables) in this paper. Section II discusses the extension of the minisuperspace by the Eisenhart-Duval lift and possible conformal invariance of the WDW equations. We study the treatment of additional degrees of freedom especially in the known simple models. In Sec. III, we propose quantum cosmology with the Dirac equation in the extended minisuperspace of the simple models, and their fundamental solutions are presented. The last section will be devoted to discussions and future prospects.

\section{MINISUPERSPACE EXTENDED BY THE EISENHART-DUVAL LIFT AND GEOMETRY OF THE WDW EQUATION}

In this paper we consider a homogeneous and isotropic universe containing a single spatially constant scalar field. We will just add comments for generic cases occasionally. First of all, we would like to review the "conventional" WDW equation, and show our approach with the Eisenhart-Duval lift afterwards.

At first, we start with the following action of the gravitating scalar field

$$
S=\int d^{4} x \sqrt{-g}\left[\frac{1}{2 \kappa^{2}} R-\frac{1}{2}(\nabla \phi)^{2}-V(\phi)\right],
$$

where $g$ is the determinant of the metric tensor $g_{\mu \nu}, R$ denotes the scalar curvature constructed from $g_{\mu \nu}(\mu, \nu=0,1,2,3),(\nabla \phi)^{2}$ means $g^{\mu \nu} \partial_{\mu} \phi \partial_{\nu} \phi$, and $V(\phi)$ represents a potential for the real scalar field $\phi$. The constant $\kappa^{2}$ equals to $8 \pi G$, where $G$ is Newton's constant.

As the metric, we assume the FLRW metric,

$$
d s^{2}=-N^{2} d t^{2}+a^{2}(t) d \Omega_{3}^{2},
$$


where $d \Omega_{3}^{2}$ denotes the maximally symmetric three-space, whose Ricci curvature ${ }^{(3)} R_{i j}$ is characterized by a constant $K$, such as ${ }^{(3)} R_{i j}=2 K g_{i j}(i, j=1,2,3)$, while $N$ is the lapse function. Assuming that the scalar field depends only on time $t$, the Lagrangian can be written in the following form ${ }^{5}$

$$
L=-\frac{1}{2 N} a \dot{a}^{2}+\frac{1}{2 N} a^{3} \dot{\phi}^{2}-N U(a, \phi)
$$

where the dot denotes the time derivative, and the potential term $U(a, \phi)$ is, for the above action (2.1),

$$
U(a, \phi)=a^{3} V(\phi)-\frac{1}{2} K a
$$

In the derivation of the Lagrangian the physical units are chosen to be $\kappa^{2}=6$, for convenience.

The canonical analysis of this action defines the Hamiltonian of the system. The lapse function $N$ plays the role of a Lagrange multiplier and we find that the Hamiltonian constraint condition $H=0$, where

$$
H=-\frac{1}{2} \frac{\Pi_{a}^{2}}{a}+\frac{1}{2} \frac{\Pi_{\phi}^{2}}{a^{3}}+U(a, \phi),
$$

with the conjugate momenta to the scale factor and the scalar field, $\Pi_{a}=-\frac{a \dot{a}}{N}$ and $\Pi_{\phi}=\frac{a^{3} \dot{\phi}}{N}$, respectively.

In quantum gravity the Hamiltonian constraint acting on states leads to a differential equation, known as the WDW equation. We interpret that the solution of the WDW equation is the physical states. Preparing a wave function $\Psi$ as a state and replacing the momenta with differential operators as

$$
\Pi_{a} \rightarrow-i \frac{\partial}{\partial a}, \quad \Pi_{\phi} \rightarrow-i \frac{\partial}{\partial \phi}
$$

we find the usual WDW equation in minisuperspace quantum cosmology [1-5]

$$
\left[\frac{1}{a^{s+1}} \frac{\partial}{\partial a} a^{s} \frac{\partial}{\partial a}-\frac{1}{a^{3}} \frac{\partial^{2}}{\partial \phi^{2}}+2 U(a, \phi)\right] \Psi(a, \phi)=0,
$$

where the constant $s$ indicates the arbitrariness in factor ordering. So far, we have obtained a description for the derivation of the conventional WDW equation.

\footnotetext{
${ }^{5}$ Here we have added the standard Gibbons-Hawking-York boundary term [39, 40].
} 
Now, let us consider an extension with a new degree of freedom. This has been proposed in previous studies $[29,30]$ as a type of the Eisenhart-Duval lift. In the present case, the lifted Lagrangian (which includes additional variable $\chi$ ) is given by ${ }^{6}$

$$
\tilde{L}=-\frac{1}{2} a \dot{a}^{2}+\frac{1}{2} a^{3} \dot{\phi}^{2}+\frac{1}{2} \frac{\dot{\chi}^{2}}{2 U(a, \phi)}=\frac{1}{2} \tilde{G}_{M N} \dot{X}^{M} \dot{X}^{N},
$$

where $X^{M}=(a, \phi, \chi)$, and the metric of the extended minisuperspace is

$$
\tilde{G}_{M N}=\operatorname{diag}\left(-a, a^{3},[2 U(a, \phi)]^{-1}\right) .
$$

Using this extended metric the Hamiltonian constraint of the system is simply written as

$$
\frac{1}{2} \tilde{G}^{M N} \tilde{P}_{M} \tilde{P}_{N}=0
$$

where the momenta $\tilde{P}_{M}$ is given by $\tilde{P}_{M}=\tilde{G}_{M N} \dot{X}^{N}$ and $\tilde{G}^{M N}$ denotes the inverse of the metric $\tilde{G}_{M N}$ as usual. This classical equation (2.10) has an apparent classical conformal invariance under $\tilde{G}_{M N} \rightarrow \Omega^{2} \tilde{G}_{M N}$ with an arbitrary function $\Omega\left(X^{M}\right)$. Here we would like to propose the following choice for extended three-dimensional minisuperspace quantum cosmology, at least because it simplifies the equation. We take the following "gauge choice", or in practice, we use the following metric of the extended minisuperspace ${ }^{7}$

$$
G_{M N}=2 U(a, \phi) \tilde{G}_{M N}=\operatorname{diag}\left(-2 U(a, \phi) a, 2 U(a, \phi) a^{3}, 1\right) .
$$

There is factor-ordering ambiguity in promoting the conjugate variables to differential operators, $P_{M} \sim-i \frac{\partial}{\partial X^{M}}$ in this case. A possible choice is to use the Laplace-Beltrami operator as the quadratic operator. In addition, we can add a term proportional to the scalar curvature $\mathcal{R}$ of the extended minisuperspace, which can appear in quantum mechanical systems. ${ }^{8}$ Consequently, the "extended" WDW equation is written by the Klein-Gordon equation in the extended minisuperspace as

$$
\left[\frac{1}{\sqrt{-G}} \partial_{M} \sqrt{-G} G^{M N} \partial_{N}-\xi \mathcal{R}\right] \Psi=0
$$

which has covariance in the extended minisuperspace. Here, $G^{M N}$ is the inverse of $G_{M N}$, $G=-(2 U)^{2} a^{4}$ is the determinant of $G_{M N}$, the derivatives are expressed as $\partial_{M} \equiv \frac{\partial}{\partial X^{M}}$, and $\xi$ is a dimensionless constant.

${ }^{6}$ The lapse function $N$ is omitted here, since the procedure is straightforward around this step.

${ }^{7}$ In the case of $n$ dimensional extended minisuperspace, we should take $G_{M N}=[2 U(a, \phi)]^{\frac{1}{n-2}} \tilde{G}_{M N}$.

${ }^{8}$ Note that the dimension of $P^{2}$ equals to that of $\hbar^{2} \mathcal{R}$. 
Here, $\mathcal{R}$ is the scalar curvature constructed from $G_{M N}$, which is defined by

$$
\mathcal{R}=G^{M N}\left(\partial_{L} \Gamma_{M N}^{L}-\partial_{M} \Gamma_{N L}^{L}+\Gamma_{M N}^{L} \Gamma_{L P}^{P}-\Gamma_{M P}^{L} \Gamma_{N L}^{P}\right)
$$

where the Christoffel symbol $\Gamma_{M N}^{L}$ is given by

$$
\Gamma_{M N}^{L}=\frac{1}{2} G^{L P}\left(\partial_{M} G_{P N}+\partial_{N} G_{P M}-\partial_{P} G_{M N}\right) .
$$

The explicit form of the extended WDW equation is written by

$$
\begin{aligned}
& \left\{\frac{1}{a^{2}} \frac{\partial}{\partial a} a \frac{\partial}{\partial a}-\frac{1}{a^{3}} \frac{\partial^{2}}{\partial \phi^{2}}\right. \\
& \left.-\left(2 a^{3} V-K a\right) \frac{\partial^{2}}{\partial \chi^{2}}+2 \xi \frac{2 a^{3}\left[\left(V^{\prime}\right)^{2}-V^{\prime \prime} V\right]+K a\left[V^{\prime \prime}-4 V\right]}{\left(2 a^{3} V(\phi)-K a\right)^{2}}\right\} \Psi(a, \phi, \chi)=0,
\end{aligned}
$$

where $V^{\prime}=\frac{\partial V}{\partial \phi}$ and $V^{\prime \prime}=\frac{\partial^{2} V}{\partial \phi^{2}}$.

If we require another constraint on a physical state

$$
-\frac{\partial^{2}}{\partial \chi^{2}} \Psi=p^{2} \Psi,
$$

the conventional WDW equation (2.7) is recovered provided that we set the constants $p^{2}=1$ and $\xi=0$. Although it sounds unhealthy to add constraints without permission, it should be noted that the WDW wave functions have been introduced intentionally for the purpose of expressing the Hamiltonian constraint in the first place. ${ }^{9}$ The specific value of $p^{2}$ is actually not significant because the scaling by a constant can be absorbed in the primary definition of the time coordinate in the FLRW metric of our universe. The interpretation of the value of $p$ will be further discussed later.

The classical equation (2.10) has conformal symmetry. Therefore, it is natural to use conformal Laplacian (Yamabe-Laplacian) when making the quantum equation. This adoption has also been suggested in the conventional minisuperspace quantum cosmology [26, 27]. Since the extended minisuperspace in the present model is three dimensional, the conformal coupling is $\xi=\frac{1}{8}$, while $\xi=\frac{n-2}{4(n-1)}$ in the case of $n$-dimensional extended minisuperspaces.

For a nonzero value of $\xi$, the WDW equation may become a different form the conventional one since the contribution from the curvature term $\mathcal{R}$ is added in general. 10 The "nice" value for $\xi$ has been discussed for quite a long time in the field of quantum field theory in

9 The two constraints (2.15) and (2.16) are compatible because they form a first-class system of constraints.

10 Note that the curvature term $\mathcal{R}$ becomes small if $K \approx 0$ and the slow-roll parameters [41] of the potential are small (i.e., $\left(\frac{V^{\prime}}{V}\right)^{2} \ll 1$ and $\frac{V^{\prime \prime}}{V} \ll 1$ ) as an inflaton potential. 
curved spacetime $[42,43]$. The value might depend on the functional measure in the path integral on manifolds [44] and we feel that ambiguity is also left despite numerous discussions with interpretation of the WDW wave function. Incidentally, the continuum limit of the d'Alembertian acting on scalar fields in causal set theory predicts the other value of the scalar curvature coupling [45-49]. It is also known that in the stochastic quantization of geodesics, a coupling term of a scalar field and the scalar curvature emerges regardless of the presence or absence of conformal symmetry $[50,51]$. There must still be many issues to be investigated on the coupling $\xi$ even in quantum cosmology in detail. The correction term of the WDW equation due to $\mathcal{R}$ itself needs to be examined more carefully for its properties, which is an important topic for the future.

There exists an interesting class of extended minisuperspace models for which the scalar curvature $\mathcal{R}$ vanishes. In such models, the extended WDW equation (2.12) with the constraint (2.16) coincides with the conventional one, regardless of the value of $\xi$ (in the case of the present gauge choice). In the present Einstein minimally-coupled scalar system, $\mathcal{R}=0$ is satisfied in the following two cases: 1) $V(\phi) \equiv 0$, i.e., free scalar case, and 2) $K=0$ and $V(\phi)=V_{0} \exp (\lambda \phi)$, where $V_{0}$ and $\lambda$ are constants, i.e., the case with a Liouville-type potential in a flat universe. It is interesting to point out that the WDW equation in each model is known to be analytically solvable $[4,52-54] .^{11}$

The WDW equations for the two models, of which extended minisuperspace is flat, are explicitly written as

(i) $\underline{\text { Model } 1}$ (the case of $V=0$ ):

$$
\left[a \frac{\partial}{\partial a} a \frac{\partial}{\partial a}-\frac{\partial^{2}}{\partial \phi^{2}}+K a^{4} \frac{\partial^{2}}{\partial \chi^{2}}\right] \Psi(a, \phi, \chi)=0,
$$

(ii) $\underline{\text { Model } 2}$ (the case of $K=0$ and $V(\phi)=V_{0} \exp \lambda \phi$ ):

$$
\left[a \frac{\partial}{\partial a} a \frac{\partial}{\partial a}-\frac{\partial^{2}}{\partial \phi^{2}}-2 a^{6} V_{0} \exp \lambda \phi \frac{\partial^{2}}{\partial \chi^{2}}\right] \Psi(a, \phi, \chi)=0 .
$$

If we additionally assume

$$
-\frac{\partial^{2}}{\partial \chi^{2}} \Psi=p^{2} \Psi
$$

we can see that each wave function satisfies the conventional WDW equation in each case, by performing the following redefinition of variables in each model

$$
\text { Model 1: } \quad \sqrt{|p|} a \rightarrow a,
$$

11 The author of Ref. [55] solved the WDW equation for the case of exponential scalar field with the dust matter. 


$$
\text { Model 2: } \quad \beta a \rightarrow a \text { and } \phi+\gamma \rightarrow \phi, \text { where } \beta^{6} e^{\lambda \gamma}=p^{2}
$$

Therefore, in these cases, the setting $p^{2}=1$ considered first is not a special one. Moreover, from the result we can obtain solutions of the extended WDW equation (even if the variable $\chi$ is not a fictitious one) as the superposition of the solutions of the conventional WDW equation under different boundary conditions. To interpret the extended WDW equation (not as a mathematical manipulation but as a fundamental one) is very interesting and may be an approach to the initial condition problem of the Universe. Note, however, that the models we found so far have no tunneling potential and we are restricted to take the wave-packet interpretation $[53,54,56-58]$ in these special cases. The analysis of the more general case will be left as a future task.

Before closing this section, we put the forms of solutions in Models 1-2 below, which will be compared with the result of the Dirac-like first-order differential equation in the extended minisuperspace introduced in the next section. For the cases, general solutions are given by superposition of the fundamental solutions $\mathcal{A}(\nu) \psi_{\nu p} e^{i p \chi}$, where $\mathcal{A}(\nu)(-\infty<\nu<\infty)$ is an appropriate amplitude.

(i) $\underline{\text { Model } 1:[4,52,53]^{12}}$

$$
\begin{array}{cc}
\psi_{\nu p}=K_{i \nu / 2}\left(\sqrt{K}|p| a^{2} / 2\right) e^{i \nu\left(\phi-\phi_{0}\right)} & (K>0), \\
\psi_{\nu p}=J_{ \pm i \nu / 2}\left(\sqrt{|K|}|p| a^{2} / 2\right) e^{i \nu\left(\phi-\phi_{0}\right)} & (K<0),
\end{array}
$$

where $\phi_{0}$ is a constant, and the functions $J_{\nu}(z)$ and $K_{\nu}(z)$ are the Bessel function and the modified Bessel function of the second kind, respectively.

(ii) Model 2:[54]

$$
\begin{array}{cc}
\psi_{\nu p}=J_{ \pm i \nu / 3}\left(i \sqrt{C}|p| e^{3 x} / 3\right) e^{i \nu\left(y-y_{0}\right)} & (C>0) \\
\psi_{\nu p}=K_{i \nu / 3}\left(i \sqrt{|C|}|p| e^{3 x} / 3\right) e^{i \nu\left(y-y_{0}\right)} & (C<0),
\end{array}
$$

where $x \equiv \alpha+\frac{\lambda}{6} \phi, y \equiv \phi+\frac{\lambda}{6} \alpha$ with $\alpha=\ln a$, and $y_{0}$ is a constant. The constant $C$ represents $2 V_{0}\left(1-\frac{\lambda^{2}}{36}\right)^{-1}$.

${ }^{12}$ For the case of $K=0$ is rather trivial (and includes no effective potential), so we do not deal with the case in this paper. 


\section{DIRAC EQUATION IN THE EXTENDED MINISUPERSPACE}

The idea of taking the square root of the WDW equation can be found in Refs. [6-11] and others. ${ }^{13}$ In previous studies, arbitrariness inevitably remains in the treatment of the potential term in the conventional WDW equation. Our treatment on how to proceed is now clear; to use the Dirac equation in the extended minisuperspace instead of the WDW equation of Klein-Gordon-type. In this case, the possible form of the equation is fixed from the covariance in the extended minisuperspace. Also note that the Dirac equation (without the mass term) has conformal covariance. ${ }^{14}$ We suppose that the gauge choice is the same as in the previous section, i.e., we adopt $G_{M N}$ as the metric of the extended minisuperspace, though there is no guarantee that the choice of the metric will commonly make the equation easy to solve.

The Dirac-like equation in the extended minisuperspace can be written down as

$$
\not D \Psi \equiv \hat{\gamma}^{M} D_{M} \Psi \equiv \gamma^{A} e_{A}^{M} D_{M} \Psi=0 .
$$

Here, the constant gamma matrices in the flat spacetime $\gamma^{A}(A=1,2,3)$ are $\gamma^{1}=\sigma^{1}, \gamma^{2}=$ $i \sigma^{2}$, and $\gamma^{3}=i \sigma^{3}$, where $\sigma^{1}, \sigma^{2}, \sigma^{3}$ are the Pauli matrices. Note that $\left\{\gamma^{A}, \gamma^{B}\right\}=-2 \eta^{A B}$, where $\eta^{A B}=\eta_{A B}=\operatorname{diag}(-1,1,1)$. The dreibein $e_{M}^{A}=\operatorname{diag}\left((2 U)^{1 / 2} a^{1 / 2},(2 U)^{1 / 2} a^{3 / 2}, 1\right)$ is defined through $\eta_{A B} e_{M}^{A} e_{N}^{B}=G_{M N}$, and $e_{A}^{M}=\operatorname{diag}\left((2 U)^{-1 / 2} a^{-1 / 2},(2 U)^{-1 / 2} a^{-3 / 2}, 1\right)$ is its inverse matrix. Accordingly, we find that $\left\{\hat{\gamma}^{M}, \hat{\gamma}^{N}\right\}=-2 G^{M N}$.

The covariant derivative $D_{M}$ for the spin connection $\omega_{M A B}$ is defined as $D_{M} \equiv \partial_{M}+$ $\frac{1}{4} \omega_{M A B} \Sigma^{A B}$, where $\Sigma^{A B} \equiv-\frac{1}{2}\left[\gamma^{A}, \gamma^{B}\right]$. The spin connection $\omega_{M A B}$ is given by

$$
\omega_{M A B}=\frac{1}{2} e_{A}^{N}\left(\partial_{M} e_{N B}-\Gamma_{M N}^{L} e_{L B}\right)-(A \leftrightarrow B) .
$$

In this section we shall specifically demonstrate the analysis of the simplest cases; Models 1 and 2 introduced in the previous section.

In Model 1 we find that the Dirac equation (3.1) is equivalent to

$$
\left[\sigma^{1}\left(a \frac{\partial}{\partial a}+1\right)+i \sigma^{2} \frac{\partial}{\partial \phi}+i \sigma^{3} \sqrt{-K} a^{2} \frac{\partial}{\partial \chi}\right] \Psi=0 .
$$

We set the two components of the wave function as

$$
\Psi=\left(\begin{array}{l}
\Psi_{+} \\
\Psi_{-}
\end{array}\right) e^{i p \chi}
$$

13 There is another example of applying supersymmetric quantum mechanics as another method for deriving first-order differential equations [13-17].

${ }^{14}$ See, for instance, Ref. [59]. 
in order to find solutions of it. Now, the equation reads in the matrix form

$$
\left(\begin{array}{cc}
-p \sqrt{-K} a^{2} & a \frac{\partial}{\partial a}+1+\frac{\partial}{\partial \phi} \\
a \frac{\partial}{\partial a}+1-\frac{\partial}{\partial \phi} & p \sqrt{-K} a^{2}
\end{array}\right)\left(\begin{array}{c}
\Psi_{+} \\
\Psi_{-}
\end{array}\right)=\left(\begin{array}{l}
0 \\
0
\end{array}\right) .
$$

The fundamental solutions of the equation are ${ }^{15}$

$$
\begin{gathered}
\Psi_{ \pm, \nu p}=\frac{1}{\sqrt{2}} e^{ \pm i \frac{\pi}{4}} K_{\frac{i \nu}{2} \mp \frac{1}{2}}\left(\sqrt{K}|p| a^{2} / 2\right) e^{i \nu\left(\phi-\phi_{0}\right)} \quad(K>0), \\
\Psi_{ \pm, \nu p}=\frac{1}{\sqrt{2}} J_{\frac{i \nu}{2} \mp \frac{1}{2}}\left(\sqrt{|K|}|p| a^{2} / 2\right) e^{i \nu\left(\phi-\phi_{0}\right)}, \pm \frac{1}{\sqrt{2}} J_{-\frac{i \nu}{2} \pm \frac{1}{2}}\left(\sqrt{|K|}|p| a^{2} / 2\right) e^{i \nu\left(\phi-\phi_{0}\right)}(K<0),
\end{gathered}
$$

where $-\infty<\nu<\infty$.

In Model 2 we find that the Dirac equation (3.1) is equivalent to

$$
\left[\sigma^{1}\left(a \frac{\partial}{\partial a}+\frac{3}{2}\right)+i \sigma^{2}\left(\frac{\partial}{\partial \phi}+\frac{\lambda}{4}\right)+i \sigma^{3} \sqrt{2 V_{0} e^{\lambda \phi}} a^{3} \frac{\partial}{\partial \chi}\right] \Psi=0 .
$$

We set $\Psi=\left(\begin{array}{c}\Psi_{+} \\ \Psi_{-}\end{array}\right) e^{i p \chi}$ and then the equation reads in the matrix form,

$$
\left(\begin{array}{cc}
-p a^{3} \sqrt{2 V_{0} e^{\lambda \phi}} & a \frac{\partial}{\partial a}+\frac{3}{2}+\frac{\partial}{\partial \phi}+\frac{\lambda}{4} \\
a \frac{\partial}{\partial a}+\frac{3}{2}-\frac{\partial}{\partial \phi}-\frac{\lambda}{4} & p a^{3} \sqrt{2 V_{0} e^{\lambda \phi}}
\end{array}\right)\left(\begin{array}{c}
\Psi_{+} \\
\Psi_{-}
\end{array}\right)=\left(\begin{array}{l}
0 \\
0
\end{array}\right) .
$$

The fundamental solutions of the equation are ${ }^{16}$

$$
\begin{gathered}
\Psi_{ \pm, \nu p}=\frac{1}{\sqrt{2}} \sqrt{1 \pm \frac{\lambda}{6}} J_{\frac{i \nu}{3} \mp \frac{1}{2}}\left(\sqrt{C}|p| e^{3 x} / 3\right) e^{i \nu\left(y-y_{0}\right)}, \pm \frac{1}{\sqrt{2}} \sqrt{1 \pm \frac{\lambda}{6}} J_{-\frac{i \nu}{3} \pm \frac{1}{2}}\left(\sqrt{C}|p| e^{3 x} / 3\right) e^{i \nu\left(y-y_{0}\right)} \\
\Psi_{ \pm, \nu p}=\frac{1}{\sqrt{2}} e^{ \pm i \frac{\pi}{4}} \sqrt{1 \pm \frac{\lambda}{6}} K_{\frac{i \nu}{3} \mp \frac{1}{2}}\left(\sqrt{|C|}|p| e^{3 x} / 3\right) e^{i \nu\left(y-y_{0}\right)}
\end{gathered}
$$

where the definition of $x$ and $y$ is the same as in the previous section.

Probability density is endowed by the square of the norm of the wave function [6-11]

$$
\text { Probability density } \propto \sqrt{|2 U|} a^{3 / 2}\|\Psi\|^{2}=\sqrt{|2 U|} a^{3 / 2}\left(\left|\Psi_{+}\right|^{2}+\left|\Psi_{-}\right|^{2}\right)
$$

since the conservation law $\partial_{M}\left(\sqrt{-G} \bar{\Psi} \hat{\gamma}^{M} \Psi\right)=0$ yields the probability density, where we set $\bar{\Psi}=\Psi^{\dagger} \gamma^{1}$. Although further discussion may be needed on the choice of the normalization, it is convenient to define $\Psi \equiv|2 U|^{1 / 4} a^{3 / 4} \Psi$ so that

$$
\text { Probability density } \propto\|\Psi\|^{2} \text {. }
$$

\footnotetext{
15 Just as in the case of second-order differential equations, there are generally two independent fundamental solutions, but solutions with regions where their absolute values are infinite are rejected.

16 Please see footnote 15 .
} 
Now, we compare the solution (3.6) of the Dirac-type equation (3.5) with the solution (2.22) of the Klein-Gordon-type equation (2.17) in Model 1 with $K>0$. Asymptotics of the modified Bessel function of the second kind with complex order are known to be [60]

$$
K_{i \frac{\nu}{2}}(y / 2) \sim \sqrt{4 \pi} e^{-\frac{\nu \pi}{4}}\left(\nu^{2}-y^{2}\right)^{-\frac{1}{4}} \sin \left(\frac{\pi}{4}-\frac{1}{2} \sqrt{\nu^{2}-y^{2}}+\frac{\nu}{2} \cosh ^{-1} \frac{\nu}{y}\right)
$$

and $[61]$

$$
\begin{array}{r}
K_{i \frac{\nu}{2} \pm \frac{1}{2}}(y / 2) \sim \sqrt{4 \pi} e^{-\frac{\nu \pi}{4} \pm i \frac{\pi}{4}}\left(\nu^{2}-y^{2}\right)^{-\frac{1}{4}}\left[\sqrt{\frac{\nu+y}{2 y}} \sin \left(\frac{\pi}{4}-\frac{1}{2} \sqrt{\nu^{2}-y^{2}}+\frac{\nu}{2} \cosh ^{-1} \frac{\nu}{y}\right)\right. \\
\left.\mp i \sqrt{\frac{\nu-y}{2 y}} \cos \left(\frac{\pi}{4}-\frac{1}{2} \sqrt{\nu^{2}-y^{2}}+\frac{\nu}{2} \cosh ^{-1} \frac{\nu}{y}\right)\right],
\end{array}
$$

as $y \rightarrow \infty$. These functions have a similar oscillatory behavior, up to a slowly changing phase shift.

According to the analysis of Kiefer [53], an arranged Gaussian wave packet tracing a common classical path

$$
a^{2}=\frac{\bar{\nu}}{\sqrt{K}|p| \cosh 2\left(\phi-\phi_{0}\right)}
$$

can be constructed with the amplitude $\mathcal{A}(\nu)$ whose center $\nu=\bar{\nu}$ takes a relatively large value in both cases of equations for Model 1, such as

$$
\mathcal{A}(\nu) \propto \frac{1}{(\sqrt{\pi} b)^{1 / 2}} e^{-(\nu-\bar{\nu})^{2} /\left(2 b^{2}\right)},
$$

where $b$ represents the width of the wave packet. The similarity in asymptotics of $K_{i \frac{\nu}{2}}$ and $K_{i \frac{\nu}{2} \pm \frac{1}{2}}$ also helps to construct wave packet solutions for the Dirac-type equation (3.9) as well as the Klein-Gordon-type equation (2.18) [54] for Model 2. Note that because the factor $\sqrt{-G} \sqrt{\left|G^{11}\right|}$ is proportional to the argument of the modified Bessel function in both models, the normalized Dirac-type wave function $\Psi$ is expressed as a superposition of $\sqrt{y} K_{i \frac{\nu}{2} \pm \frac{1}{2}}(y)$.

The different behaviors of the functions $K_{i \frac{\nu}{2}}(y / 2)$ and $\sqrt{y / 8} K_{i \frac{\nu}{2} \pm \frac{1}{2}}(y / 2)$ seems to be especially around $y=0$. Therefore, the difference between wave-packet solutions of equations of Klein-Gordon-type (constructed from of $\psi_{\nu p}$ ) and Dirac-type (constructed from $\left.\Psi_{ \pm, \nu p} \equiv\left|2 U a^{3}\right|^{1 / 4} \Psi_{ \pm, \nu p}\right)$ is expected to be found in the region of small scale factors.

In this section, we have implicitly accepted the condition $p=$ const. As stated in Sec. II, we can consider $\chi$ as a real coordinate and can also take the wave function which has nontrivial dependence on $\chi$. It will be, however, the subject to future research. 


\section{DISCUSSION AND OUTLOOK}

In this paper, the WDW equation has been reformulated as a partial differential equation with the Laplacian defined in a minisuperspace extended by the Eisenhart-Duval lift. We have also obtained the wave function as an exact fundamental solution to the Dirac equation in the extended minisuperspace of specific models. We should emphasize that few papers have evaluated the concrete form of the solutions so far. Further research is needed for general cosmological models. It is important to study the geometrical property of the extended minisuperspace and the possibility of geometrical classification on whether the model can be solved or not. We wish to properly understand the handling of additional degrees of freedom and the degree of conformal transformation (and its possible extended symmetry) from various standpoints, as well as the straightforward generalization to the quantum cosmology of modified gravities. In any case, the present framework based on the extended minisuperspace creates many new research agendas.

Here we consider general dimensional cases with conformal coupling in the WDW equation. As a special case, we can imagine a case that the extended minisuperspace is conformally flat. Provided that the extended minisuperspace is conformally flat, the curvature $\mathcal{R}$ will be zero if the appropriate conformal transformation, or gauge, is chosen. Therefore, in this case, the WDW equation is described as a normal Laplace equation.

The chosen gauge (2.11) at the classical level is a gauge that enables the simple interpretation when the expanded minisuperspace is three dimensional and conformally flat. If the extended minisuperspace is three dimensional, the necessary and sufficient condition for conformally flatness is that the Cotton tensor is zero [62]. Under the gauge choice (2.11), if the minisuperspace is conformally flat it can be shown that the (Lorentzian) subspace stretched by variables $a$ and $\phi$ is a space with constant scalar curvature [63, 64]. Therefore, the scalar curvature $r$ of the three-dimensional minisuperspace is a constant. Since the gauge $G_{\chi \chi}=1$ is chosen, the equation becomes the WDW equation in which the coefficient before the potential term is $p^{2}+\xi r$, instead of $p^{2}$ as in our previous analysis. In our two models examined in the present paper, the value of $r$ happened to be zero. Although we have not yet found an example of a model with nonzero $r$, the model with three-dimensional extended minisuperspace of conformally constant curvature belongs to an interesting class of quantum cosmological models. 
Another example of a flat three-dimensional extended minisuperspace with the gauge $G_{\chi \chi}=1$ is the system with a general nonminimally [65] (including conformal [2, 52, 57, $58,66]$ ) coupled scalar field without any scalar potential term (including the cosmological term) in the spacetime with nonvanishing spatial curvature. ${ }^{17}$ However, in general cases, the WDW equation may be solved only when the variable is far from the original variable, which may rather make the analysis difficult. Generally speaking, including this example, solvability of models obtained by the Eisenhart-Duval lift is technically another important problem, and we would like to classify the cases skillfully in future work.

It should be pointed out that, since the equations obtained in the present paper are conventional forms of the Laplacian operator and the Dirac operator which appear in field theories, it is natural to bring them to the third quantization $[8,71-77]$ with and without introducing the non-linear term of the wave function. We can speculatively imagine some nonlinear Schrödinger equations or the Lane-Emden type equations in the (extended) minisuperspace. In the context of the third quantization, it is also interesting to speculate that the global structure of the extended minisuperspace, such as the compactness of the manifold, will affect the quantum dynamics of the Universe.

On the other hand, we would like to consider the formulation using the Eisenhart-Duval metric with more degrees of freedom [31,33-35] in quantum cosmology. In addition, although we have focused on the discussion in the minisuperspace so far, we hope that we can proceed with the Eisenhart-Duval lift in more basic quantum field theory of gravity ${ }^{18}$ with reference to previous research $[80,81]$.

\section{ACKNOWLEDGMENTS}

The authors are grateful to P. Horváthy for important information and references. The authors thank A. Paliathanasis for a reference and information on an interesting recent study

\footnotetext{
17 The extended minisuperspaces for the Kaluza-Klein theory with a cosmological constant [67, 68], the scalar-tensor theory with a cosmological constant [69], and the Kantowski-Sachs model with a cosmological constant [70], which are all known to be related to the similar model, are also flat.

18 The geodesics and the quantum Hamiltonian in the infinite-dimensional superspace have been investigated
} $[78,79]$. 
[82]. The authors also thank J. C. Feng for old and new references.

[1] J. B. Hartle and S. W. Hawking, "Wave function of the universe", Phys. Rev. D28 (1983) 2960.

[2] S. W. Hawking, "The quantum state of the universe", Nucl. Phys. B239 (1984) 257.

[3] J. J. Halliwell, "Introductory lectures on quantum cosmology", in: Proceedings of 7th Jerusalem Winter School for Theoretical Physics: Quantum Cosmology and Baby Universes, edited by S. Coleman, J. B. Hartle, T. Piran and S. Weinberg (World Scientific, Singapore, 1991), pp. 159-243. arXiv:0909.2566 [gr-qc].

[4] C. Kiefer, Quantum Gravity, Int. Ser. Monogr. Phys. Vol. 155 (Clarendon Press, Oxford, 2012).

[5] C. Kiefer, "Conceptual problems on quantum gravity and quantum cosmology", ISRN Mathematical Physics 2013 (2013) 509316. arXiv:1401.3578 [gr-qc].

[6] P. D. D'Eath, S. W. Hawking and O. Obregón, "Supersymmetric Bianchi models and the square root of the Wheeler-DeWitt equation", Phys. Lett. B300 (1993) 44.

[7] C.-M. Kim and S.-K. Oh, "Dirac-square-root formulation of some types of minisuperspace quantum cosmology", J. Korean Phys. Soc. 29 (1996) 549.

[8] Y.-G. Shen and Z.-Y. Cheng, "Quantization of the Bianchi type universe", Chin. Astron. Astrophys. 21 (1997) 389.

[9] H. Yamazaki and T. Hara, "Dirac decomposition of Wheeler-DeWitt equation in the Bianchi class A models", Prog. Theor. Phys. 106 (2001) 323. gr-qc/0101066.

[10] S. A. Hojman and F. A. Asenjo, "Supersymmetric Majorana quantum cosmologies", Phys. Rev. D92 (2015) 083518. arXiv:1506.02939 [gr-qc].

[11] C. A. Rubio, F. A. Asenjo and S. A. Hojman, "Quantum cosmologies under geometrical unification of gravity and dark energy", Symmetry 11 (2019) 860.

[12] A. Mostafazadeh, "Two-component formulation of the Wheeler-DeWitt equation", J. Math. Phys. 39 (1998) 4499. gr-qc/9610012.

[13] R. Graham, "Supersymmetric Bianchi type-IX cosmology", Phys. Rev. Lett. 67 (1991) 1381.

[14] R. Graham, "Supersymmetric general Bianchi type IX cosmology with a cosmological term", Phys. Lett. B277 (1992) 393. 
[15] O. Obregón, J. Socorro and J. Benítez, "Supersymmetric quantum cosmology proposals and the Bianchi type-II model", Phys. Rev. D47 (1993) 4471.

[16] O. Obregón, J. Pullin and M. P. Ryan, "Bianchi cosmologies: New variables and a hidden supersymmetry", Phys. Rev. D48 (1993) 5642. gr-qc/9308001.

[17] C. Ramírez and V. Vázquez-Báez, "Quantum supersymmetric FRW cosmology with a scalar field", Phys. Rev. D93 (2016) 043505. arXiv:1511.04425 [gr-qc].

[18] F. Cooper, A. Khare and U. Sukhatme, "Supersymmetry and quantum mechanics", Phys. Rep. 251 (1995) 267. hep-th/9405029.

[19] P. Hajicek, "Origin of nonunitarity in quantum gravity", Phys. Rev. D34 (1986) 1040.

[20] C. Simeone, "Quantization of minisuperspaces as ordinary gauge systems", J. Math. Phys. 39 (1998) 3131. gr-qc/0108080.

[21] G. Catren and R. Ferraro, "Quantization of the Taub model with extrinsic time", Phys. Rev. D63 (2000) 023502. gr-qc/0006027.

[22] V. E. Kuzmichev and V. V. Kuzmichev, "The big bang quantum cosmology: The matterenergy production epoch", Acta Phys. Pol. 39 (2008) 979. arXiv:0712.0464 [gr-qc].

[23] N. Kan, M. Kuniyasu, K. Shiraishi and K. Takimoto, "Equivalent Hamiltonian approach to quantum cosmology of integrable models", Class. Quant. Grav. 37 (2020) 105002. arXiv:1908.09286 [gr-qc].

[24] N. C. Tsamis and R. P. Woodard, "The factor-ordering problem must be reglated", Phys. Rev. D36 (1987) 3641.

[25] S. W. Hawking and D. N. Page, "Operator ordering and the flatness of the universe", Nucl. Phys. B264 (1986) 185.

[26] I. Moss, "Quantum cosmology and the self observing universe", Ann. Inst. Henri Poincaré 49 (1988) 341.

[27] J. J. Halliwell, "Derivation of the Wheeler-DeWitt equation from a path integral for minisuperspace models", Phys. Rev. D38 (1988) 2468.

[28] N. Kontoleon and D. L. Wiltshire, "Operator ordering and consistency of the wave function of the Universe", Phys. Rev. D59 (1999) 063513. gr-qc/9807075.

[29] K. Finn and S. Karamitsos, "Finite measure for the initial conditions of inflation", Phys. Rev. D99 (2019) 063515. arXiv:1812.07095 [gr-qc]. 
[30] K. Finn, "Initial conditions of inflation in a Bianchi I universe", Phys. Rev. D101 (2020) 063512. arXiv:1912.04306 [gr-qc].

[31] L. P. Eisenhart, "Dynamical trajectories and geodesics", Ann. Math. 30 (1928) 591.

[32] C. Duval, G. Burdet, H. P. Künzle and M. Perrin, "Bargmann structures and Newton-Cartan theory", Phys. Rev. D31 (1985) 1841.

[33] L. Casetti, M. Pettini and E. G. O. Cohen, "Geometric approach to Hamiltonian dynamics and statistical mechanics", Phys. Rep. 337 (2000) 237. cond-mat/9912092.

[34] M. Pettini, Geometry and topology in Hamiltonian dynamics and statistical mechanics (Springer, New York, 2007)

[35] M. Cariglia, "Hidden symmetries of dynamics in classical and quantum physics", Rev. Mod. Phys. 86 (2014) 1283. arXiv:1411.1262 [math-ph].

[36] S. Dhasmana, A. Sen and Z. K. Silagadze, "Equivalence of a harmonic oscillator to a free particle and Eisenhart lift", Ann. Phys. (NY) 434 (2021) 168623. arXiv:2106.09523 [quantph].

[37] M. Cariglia, A. Galajinsky, G. W. Gibbons and P. A. Horvathy, "Cosmological aspects of the Eisenhart-Duval lift", Eur. Phys. J. C78 (2018) 314. arXiv:1802.03370 [gr-qc].

[38] C. Duval, G. Gibbons and P. Horváthy, "Celestial mechanics, conformal structures, and gravitational waves", Phys. Rev. D43 (1991) 3907. hep-th/0512188.

[39] J. W. York, "Role of conformal three-geometry in the dynamics of gravitation", Phys. Rev. Lett. 28 (1972) 1082.

[40] G. W. Gibbons and S. W. Hawking, "Action integrals and partition functions in quantum gravity", Phys. Rev. D15 (1977) 2752.

[41] J. Ellis and D. Wands, "23. Inflation", in: P. A. Zyla et al. (eds.) Review of Particle Physics (Particle Data Group), Prog. Theor. Exp. Phys. 2020 (2020) 083C01.

[42] L. Parker, "Path integrals for a particle in curved space", Phys. Rev. D19 (1979) 438.

[43] J. D. Beckenstein and L. Parker, "Path-integral evaluation of Feynman propagator in curved space", Phys. Rev. D23 (1981) 2850.

[44] T. Laetsch, "An approximation to Wiener measure and quantization of the Hamiltonian on manifolds with non-positive sectional curvature", J. Funct. Anal. 265 (2013) 1667. arXiv:1210.3524 [math.PR]. 
[45] D. M. T. Benincasa and F. Dowker, "Scalar curvature of a causal set", Phys. Rev. Lett. 104 (2010) 181301. arXiv:1001.2725 [gr-qc].

[46] F. Dowker and L. Glaser, "Causal set d'Alembertians for various dimensions", Class Quant. Grav. 30 (2013) 195016. arXiv:1305.2588 [gr-qc].

[47] L. Glaser, "A closed form expression for the causal set d'Alembertian", Class Quant. Grav. 31 (2014) 095007. arXiv:1311.1701 [math-ph].

[48] A. Belenchia, "Universal behaviour of generalized causal set d'Alembertian in curved spacetime", Class Quant. Grav. 33 (2016) 135011. arXiv:1510.04665 [gr-qc].

[49] A. Belenchia, D. M. T. Benincasa and F. Dowker, "The continuum limit of a four-dimensional causal set scalar d'Alembertian", Class Quant. Grav. 33 (2016) 245018. arXiv:1510.04656 $[\mathrm{gr}-\mathrm{qc}]$.

[50] F. Kuipers, "Stochastic quantization on Lorentzian manifolds", JHEP 2105 (2021) 028. arXiv:2101.12552 [hep-th].

[51] F. Kuipers, "Stochastic quantization on Relativistic Theories", arXiv:2103.02501 [gr-qc].

[52] S. W. Hawking and D. N. Page, "Spectrum of wormholes", Phys. Rev. D42 (1990) 2655.

[53] C. Kiefer, "Wave packets in minisuperspace", Phys. Rev. D38 (1988) 1761.

[54] A. A. Andrianov, C. Lan, O. O. Novikov and Y.-F. Wang, "Integrable Cosmological Models with Field: Energy Density Self-Adjointness and Semiclassical Wave Packets", Eur. J. Phys. C78 (2018) 786. arXiv:1802.06720 [hep-th].

[55] A. Paliathanasis, "Dust fluid component from Lie symmetries in scalar field cosmology", Mod. Phys. Lett. A32 (2017) 1750206. arXiv:1710.08666 [gr-qc].

[56] Y. Kazama and R. Nakayama, "Wave packet in quantum cosmology", Phys. Rev. D32 (1985) 2500.

[57] C. Kiefer, "Wave packets in quantum cosmology and the cosmological constant", Nucl. Phys. B341 (1990) 273.

[58] S. S. Gousheh and H. R. Sepangi, "Wave packets and initial conditions in quantum cosmology", Phys. Lett. A272 (2000) 304. gr-qc/0006094.

[59] O. Hijazi, "A conformal lower bound for the smallest eigenvalue of the Dirac operator and Killing spinors", Commun. Math. Phys. 104 (1986) 151.

[60] A. Erdélyi,W. Magnus, F. Oberhettinger and F. G. Tricomi, Higher Transcendental Functions, vol. 2 (McGraw-Hill, New York, 1953). 
[61] J. Tseng, "Eisenstein series and an asymptotic for the $K$-Bessel function", Ramanujan J. 56 (2021) 323. arXiv:1812.09450 [math.NT].

[62] É. Cotton, "Sur les variétés à trois dimensions", Ann. Fac. d. Sc. Toulouse (II) 1 (1899) 385.

[63] D. Grumiller and R. Jackiw, "Kaluza-Klein reduction of conformally flat spaces", Int. J. Mod. Phys. D15 (2006) 2075. math-ph/0609025.

[64] R. Jackiw, "Dimensional reduction of conformal tensors and Einstein-Weyl spaces", SIGMA 3 (2007) 091. arXiv:0708.3788 [math-ph]

[65] C. Kiefer, "Non-minimally coupled scalar fields and the initial value problem in quantum gravity", Phys. Lett. B225 (1989) 227.

[66] P. Pedram, "On the conformally coupled scalar field quantum cosmology", Phys. Lett. B671 (2009) 1. arXiv:0811.3668 [gr-qc].

[67] J. Wudka, "Quantum effects in a model of cosmological compactification", Phys. Rev. D35 (1987) 3255.

[68] J. Wudka, "Boundary conditions and the cosmological constant", Phys. Rev. D36 (1987) 1036.

[69] J. E. Lidsey, "Scale factor duality and hidden supersymmetry in scalar-tensor cosmology", Phys. Rev. D52 (1995) R5407. gr-qc/9510017.

[70] H.-D. Conradi, "Remarks on Kantowski-Sachs quantum cosmology", Nucl. Phys. B (Proc. Suppl.) 57 (1997) 295.

[71] N. Caderni and M. Martellini, "Third quantization formalism for Hamiltonian cosmologies", Int. J. Theor. Phys. 23 (1984) 233.

[72] M. McGuigan, "Third quantization and the Wheeler-DeWitt equation", Phys. Rev. D38 (1988) 3031.

[73] M. McGuigan, "Universe creation from the third-quantized vacuum", Phys. Rev. D39 (1989) 2229 .

[74] A. Hosoya and M. Morikawa, "Quantum field theory of the Universe", Phys. Rev. D39 (1989) 1123.

[75] Y. Ohkuwa, Y. Ezawa and M. Faizal, "Constraints on operator ordering from third quantization”, Ann. Phys. (NY) 365 (2016) 54. arXiv:1505.02754 [physics.gen-ph].

[76] Y. Ohkuwa, Y. Ezawa and M. Faizal, "Operator ordering ambiguity and third quantization", Ann. Phys. (NY) 414 (2020) 168072. arXiv:1907.02042 [hep-th]. 
[77] S. J. Robles-Pérez, "Quantum cosmology in the light of quantum mechanics", Galaxies 7 (2019) 50. arXiv:1812.10657 [gr-qc].

[78] B. S. DeWitt, "Spacetime as a sheaf of geodesics in superspace", in: Relativity: Proceedings, edited by M. Carmeli, S. I. Fickler and L. Witten (Springer, Boston, 1970), pp. 359-374.

[79] J. C. Feng and R. A. Matzner, "From path integrals to the Wheeler-DeWitt equation: Time evolution in spacetimes with a spatial boundary", Phys. Rev. D96 (2017) 106005. arXiv:1706.02431 [physics.class-ph].

[80] K. Finn, S. Karamitsos and A. Pilaftsis, "Eisenhart lift for field theories", Phys. Rev. D98 (2018) 016015. arXiv:1806.02431 [physics.class-ph].

[81] K. Finn, S. Karamitsos and A. Pilaftsis, "Quantizing the Eisenhart lift", Phys. Rev. D103 (2021) 065004. arXiv:2012.15288 [hep-th].

[82] A. Zampeli and A. Paliathanasis, "Quantization of inhomogeneous spacetimes with cosmological constant term", Class. Quant. Grav. 38 (2021) 165012. arXiv:2012.10814 [gr-qc]. 